\title{
Jouer avec la forme : politique et poétique du jazz dans la prose et les chansons de Boris Vian
}

\author{
Olivier BOURDERIONNET (New Orleans)
}

\section{Summary}

This article examines the multi-faceted influence of jazz music on Boris Vian's writing process. As a jazz critic, a songwriter and a novelist, Vian did not merely write about jazz or use it as a prop. His writing displays a political conscience in tune with class and race issues as well as a strong measure of audacity and irreverence that characterized the music of the jazz artists he most admired. A closer look at his activity as a singer-songwriter and an analysis of his formal approach in chapter 32 of L'écume des jours reveal a disdain for the conventional and point to traces of Vian's own musical practice transposed to the page.

Une fréquentation, même superficielle de l'œuvre foisonnante de Boris Vian mène, tôt ou tard, à une rencontre avec le jazz. L'omniprésence de cette musique dans son œuvre et notamment dans son roman le plus connu L'écume des jours (1947) a fait l'objet de commentaires passionnants dans les travaux de Gilbert Pestureau, Noël Arnaud, JeanLouis Pautrot, Akiko Fukagawa, et plus récemment Cécile Pajona. Les critiques ayant examiné L'écume des jours sous l'angle du jazz ont insisté sur son influence esthétique et structurelle ainsi que sur son rôle central en tant que protagoniste du roman. En effet, des références discographiques, aux décors et motifs musicaux, en passant par l'expérimentation prosodique et la synesthésie, les registres dans lesquels se manifeste l'intertexte jazzistique sont multiples. En tentant d'apporter une modeste pierre à un édifice critique déjà important, la présente étude, se penchera sur ce même ouvrage afin d'en illustrer un nouvel aspect d'expérimentation formelle qui entre dans le cadre de l'auto-référence et de la private joke, mais elle se propose également d'étendre un regard sur le rôle du jazz chez Vian comme moteur de gestes esthétiques et politiques, particulièrement dans les Chroniques de jazz et dans les Chansons possibles et impossibles, interprétées par lui et enregistrées sous son nom. 


\section{Critique de jazz et climat politique}

Le jeune Boris Vian découvre le jazz à l'adolescence, vers 1935, et décide, peu après, de se consacrer à la trompette. L'écriture de chansons, qu'il pratique alors sous forme de pastiche, pour des spectacles montés avec ses amis de lycée, et la musique de jazz, précèdent ainsi les expériences romanesques. Son premier conte, Trouble dans les Andains, ne sera écrit qu'aux environs de 1942. De cette rencontre fondatrice avec le jazz, nous nous proposons donc d'examiner les échos et les répercussions en gestes politiques et créateurs, et ce, dans trois activités distinctes de Vian, celle du critique, celle de l'auteur-interprète de chanson, et enfin celle du romancier.

Dans son "Avant propos " aux célèbres Chroniques de jazz éditées par ses soins en $1967^{1}$ le critique de jazz, auteur et professeur Lucien Malson, collègue de Vian à Jazz Hot, propose la remarque suivante :

Le jazz était chez Boris Vian [...] la plus chère des préoccupations, si Vian romancier se désintéressera un jour des romans, Vian critique aimera le jazz jusqu’à sa mort. Ce qui frappe dans l'aventure d'un homme aux vocations innombrables, c'est la constance, la permanence, l'immuabilité de cette passion. La musique négro-américaine ne fut pas pour lui comme pour beaucoup d'intellectuels de sa génération, un art > intéressant parmi d'autres. (Vian 1971, 8)

Pour Malson, Vian se distingue clairement de la catégorie des écrivains qui se mêlent de jazz afin de sacrifier à une mode ou à un engouement passager. À l'époque où Vian débute sa collaboration avec le magazine Jazz Hot, en 1946, il est ingénieur à l'AFNOR (Agence Française de Normalisation) et fait partie depuis cinq ans (avec son frère Alain Vian, qui tient la batterie) de l'orchestre de jazz de Claude Abadie. Un orchestre s semi-amateur > qui anime des soirées dansantes et se produit régulièrement pour des soldats américains au Rainbow Corner, place de la Madeleine. Ses contributions critiques à Jazz Hot, quasi bénévoles, sous forme de revues de presse, interviennent dans une période où les publications spécialisées et les clubs d'aficionados répartis sur tout le territoire français et en Europe, se passionnent pour les concerts d'artistes américains en tournée, les éditions de nouveaux enregistrements, et s'égarent dans d'interminables querelles d'écoles débutées pour certaines avant-guerre (Lane 2013, 91). Ainsi que l'a bien analysé Elizabeth Vihlen McGregor dans son récent ouvrage Jazz and Postwar French Identity, dans les deux décennies qui ont suivi la Seconde Guerre mondiale, la critique de jazz a été le lieu de prises de positions idéologiques auxquelles le contexte géopolitique de la guerre froide et de la décolonisation n'était pas étranger. ${ }^{2}$ Une partie de cette critique visait inévitablement les États-Unis et leur politique ségrégationniste. McGregor insiste sur la propension de cette critique française de jazz à pointer du doigt la politique étatsunienne vis-à-vis de sa population afro-américaine tout en ignorant la violence de la politique coloniale de la France en Algérie. Vian, dont elle ne mentionne pas le travail de critique mais dont la sévérité envers les États-Unis s'exprime régulièrement 
dans ses articles sur l'actualité du jazz, a pour sa part mis en évidence le parallèle entre ces deux manifestations d'injustice notamment dans le numéro de Jazz Hot de juin 1956, au sujet d'un article de Nat Hentoff portant sur une pratique de détournement des droits d'auteurs à laquelle se livrent certains musiciens de jazz américains en transformant les titres et la mélodie de morceaux célèbres (mais en en conservant la structure harmonique) pour les déposer sous leur nom. Vian fait une analogie avec une pratique répandue à la radio et dans les salles de danse, sous l'Occupation, qui consistait à donner des titres alternatifs à des morceaux de musique américaine pour, soi-disant, contourner la censure mise en place par les autorités allemandes. Il fait alors une référence indirecte, à la situation algérienne :

Effectivement, il y a pas mal de droits qui se perdent pour le compositeur, dans ce genre de coups. Ça me rappelle le bon vieux temps - vous savez, l'occupation, quand on était les Algériens des Allemands - où l'on rebaptisait "Lady Be Good " "Les Bigoudis " pour ennuyer M. Goebbels. Ah, là là, on était jeunes... (Vian 1971, 126)

Les références sont rares, à ce qu'on appelait à l'époque les ‘ événements en Algérie, dans les revues de presse que Vian signe pour Jazz Hot. L'exercice se prête assez mal au commentaire de l'actualité politique. Vian prend avant tout position sur ce qui concerne la politique du jazz, ne manquant jamais de mettre en évidence les situations aberrantes causées par les excès des syndicats de musiciens anglais, par exemple. Il se permet également de traiter un journaliste lyonnais de "suppôt de Truman, de valet du capitalisme nazi » (Vian 1971, 409). En revanche, contrairement à ce que l'on a pu reprocher à d'autres critiques trop heureux de mettre en avant le racisme anti-Noirs en Amérique et ignorant le racisme environnant, les revues de presse de Vian épinglent régulièrement les expressions de racisme ordinaire chez ses concitoyens. À propos de décisions administratives discriminatoires à l'encontre du jazz et des Afro-Américains dans la ville de Memphis, il signale les abus dont se rendent coupables les agents de police français à l'occasion d'un concert de Dizzy Gillespie à Paris. Il écrit :

La police de tous les pays est décidément en bonne voie. Il n’y a pas si longtemps, en effet, qu'au concert de Dizzy Gillespie à Pleyel, des agents repoussaient à coups de poings les amateurs d'autographes en s'étonnant que des ‘ civilisés ‘ puissent s'intéresser à cette ‘ musique de sauvages > [...] si les choses ont un peu plus évolué ici, il n’en reste pas moins qu'au même concert de Gillespie des spectateurs ont crié "À Tombouctou !" et on lançait à Dizzy l'apostrophe suivante: «Va apprendre le français, eh, mal blanchi ! » (Vian 1971, 86)

Ailleurs, il met en évidence les commentaires à caractère raciste dans Le journal de la femme. On est frappé par la ressemblance avec le discours répandu de l'extrême droite française dans les années 1920 et 1930 , assimilant le jazz à une pathologie ${ }^{3}$ : 
Vous ne connaissez pas le be-bop ? [...] chacun des membres joue n'importe quoi sans s'occuper des autres et [que] l'harmonie qui en résulte doit être comparable à une troupe de singes s'emparant des instruments, pianotant, soufflant et tapant au hasard de leur inspiration [...] mais il n'empêche que le be-bop gagne du terrain ce qui n'est pas pour nous surprendre à notre époque de folie où la fausse note et l'incohérence règnent partout. LES HOMMES POLITIQUES CHEZ NOUS ET AILLEURS NE SEMBLENT-ILS PAS DES PARTISANS FANATIQUES DU BE-BOP ? (Vian 1971, 392 ; majuscules de Vian)

Mois après mois, les revues de presse de Vian font apparaître deux grandes constantes qui semblent préoccuper les acteurs du jazz, les amateurs, producteurs, journalistes, ainsi que certains musiciens. La première concerne la supériorité des orchestres américains sur les orchestres européens et australiens. Les enjeux en sont importants car ils concernent l'emploi des musiciens mais aussi la capacité à juger des qualités créatives des uns et des autres. Vian ne manque pas de manifester son exigence et son impatience vis à vis de certains orchestres américains qui tombent dans la facilité mais jouissent de conditions avantageuses dans les villes européennes. Toutefois, il maintient que les musiciens américains demeurent les véritables inventeurs du jazz et que les Européens (ces critiques paraissent dans les années quarante et cinquante) font, au mieux, office de bons élèves. La seconde constante qui divise de façon plus marquée encore, concerne la question du bebop comme le signe d'une décadence du jazz. L'école new-yorkaise de la $52^{\text {ème }}$ rue est violemment rejetée par un front critique mené par Hugues Panassié (fondateur du Hot Club de France) qui refuse de considérer le bebop comme du jazz. Il n'accepte que la musique jouée dans une certaine tradition et par des musiciens noirs. Vian, comme Charles Delaunay, est en profond désaccord avec Panassié à propos du bebop dont Dizzy Gillespie et Charlie Parker sont les chefs de file. Mais il partage son opinion pour ce qui concerne la supériorité des musiciens noirs sur les musiciens blancs, ainsi que la plupart des critiques français de l'époque dont André Hodeir par exemple.

Sur cette question, Vian affiche, lui aussi une position catégorique. Il ne manque jamais de rappeler que le jazz a été inventé par les Afro-Américains « le jazz a tout de même la peau noire... ne pas oublier» (Vian 1971, 147) que ceux-ci ont donc un droit sur cette musique, et que les orchestres blancs, en général, ne proposent qu'une forme de jazz sans saveur dépourvue des qualités essentielles du genre. Si Vian est capable de reconnaître des qualités à certains instrumentistes blancs (à Bix Beiderbecke, par exemple) il ne cache jamais sa préférence pour les artistes afro-américains. Ses cibles préférées étant le chef d'orchestre blanc Stan Kenton et le pianiste Dave Brubeck : "Je maintiens, puisque je suis raciste, que jamais les Blancs n'égaleront les Noirs en matière de jazz, je m'excuse de répéter ici ce que j’ai déjà écrit dans Combat mais je le pense encore. "(Vian 1971, 149) À travers la loyauté indéfectible de Vian vis-à-vis des immenses artistes que sont Louis Armstrong, Sydney Bechet, Duke Ellington, Billie Holiday, Ella Fitzgerald, Charlie Parker, Miles Davis et d'autres, s'exprime une prise de position politique qu'il faut bien évidemment replacer dans le contexte qui précède l'intensification de la lutte de la communauté afro-américaine pour l'obtention des droits 
civiques. Aux États-Unis, durant les années trente et quarante, la multiplication d'orchestres blancs de swing a contribué à répandre une musique de jazz édulcorée, au détriment des musiciens noirs qui n'ont pas accès à la manne d'emplois d'orchestres radiophoniques, de cinéma et de salles de spectacle, et n'exercent aucun contrôle dans l'industrie du disque. Cette visibilité blanche du jazz a encouragé le phénomène de ‘ color blindness > perpétuant la discrimination dans le monde de la presse et du spectacle au travers d'une forme de racisme muet qui feint d'ignorer la situation et se donne occasionnellement bonne conscience en accueillant favorablement de rares orchestres interraciaux (Panish 1995, 10). Ce terme de ‘ racisme ' que Vian revendique à son propre égard, ne s'explique donc pas uniquement par l'essentialisme généralisé dans la critique française de l'époque mais s'explique également comme une posture militante en réaction aux ravages de la < color blindness ). Il a - aux yeux de Vian - pour objectif de sauver l'intégrité du jazz et de restituer aux musiciens AfroAméricains le respect et l'honneur qui leur revient avec l'espoir de sensibiliser le public à une situation qui perdure. Ce « racisme > revendiqué de Vian trouve donc également son origine dans un sentiment d'injustice, un désir de réparation devant la situation des musiciens afroaméricains auxquels il donne la priorité qui leur revient dans sa revue de presse des années quarante et cinquante.

\section{Le jazz pour penser la chanson autrement}

En tant qu'auteur d'une revue de presse sur le jazz, la contribution créative et politique de Vian s'inscrit dans le monde du jazz au sens beckerien du terme qui insiste sur la notion (interactive > et attribue à tous les acteurs d'un champ artistique leur part de responsabilité et de mérite, dans la production des œuvres. En effet, pour le sociologue Howard Becker (pianiste de jazz lui-même) l'art est le résultat d'un travail collectif qui met en œuvre une myriade de compétences. Dans cette optique, il faut juxtaposer la contribution de Vian musicien avec celle de Vian critique, animateur au Tabou puis au Club St. Germain où il accueille des musiciens de l'avant-garde, réalisateur et hôte d'émissions radiophoniques sur le jazz, directeur artistique, concepteur de pochettes de disques, et bien sûr d'auteur-interprète de chansons. C'est vers cette dernière activité que nous allons à présent nous tourner, tant la production de Vian, interprète de ses propres chansons, se signale avec évidence comme un autre domaine où s'expriment les forces conjuguées d'une esthétique jazz et d'un engagement politique. Le choix de la présentation de ses deux disques 45 tours Chansons « impossibles » d'abord, puis Chansons "possibles ", enregistrés en avril 1955, annonce, d'emblée, le caractère non-conventionnel du produit et cherche à attirer l'attention sur l'aspect subversif de ses chansons pour transformer celui-ci en un argument commercial. Rappelons que dans la même maison de disques Philips, le genre provocateur vient d'être lancé, quelques années auparavant, par Georges Brassens qui mobilise la censure morceau après morceau, et que le disque de Mouloudji chantant "Le déserteur " écrit et composé par Vian vient également d'être censuré, malgré des retouches sur la fin du texte finalement consenties par ce dernier. 
Alors que la guerre d'Indochine est à peine terminée et que la guerre d'Algérie ne dit pas encore son nom, l'antimilitarisme des quatre chansons "impossibles ", "Les joyeux bouchers », « Le déserteur », « Le petit commerce », « La java des bombes atomiques », frappe par l'ironie et le ton satirique qui les traversent. L'autorité de l'État y est ridiculisée, des hommes de pouvoir sans humanité y sont comparés à des marchands de canons ou à des directeurs d'abattoirs. En guise d'introduction au "Petit commerce "Vian lance une pique au fameux député populiste Pierre Poujade : "Pour consoler Monsieur Poujade, l'histoire d'un artisan qui a réussi ». Parmi les chansons " possibles » : « La complainte du progrès ", "Le cinématographe ", " J'suis snob " et " On n'est pas là pour se faire engueuler », seule la seconde " Le cinématographe » échappe à la satire sociale bien qu'on y sente déjà l'annonce de la critique du spectacle telle qu'elle sera bientôt articulée dans le premier numéro de Linternationale situationniste notamment dans l'article "Avec et contre le cinéma " (Internationale Situationniste 1958, 8). Les trois autres titres, proposent une critique mordante de la société de consommation, d'un État militaire répressif, et ridiculisent les pratiques de distinction sociales des classes privilégiées (dont Vian est lui-même originaire).

Les deux 45 tours ont été enregistrés avec l'orchestre et les arrangements de Jimmy Walter qui est aussi le compositeur de la plupart des musiques. Les compétences des musiciens et de l'arrangeur en matière de jazz apparaissent dans la plupart des plages où se mélangent clarinettes, batterie, banjo, guitare, piano, trompettes, saxophones et trombones. Cependant, on ne peut parler d'une véritable dominance du jazz si ce n'est sur " J'suis snob ", "Le cinématographe » et "Le petit commerce ». Les autres musiques et arrangements de Jimmy Walter mêlent le jazz à d'autres styles comme la marche, le tango ou encore des rythmes d'inspiration afro-cubaine. Cependant, l'écriture des arrangements par son côté inventif, non-conventionnel et parfois comique, se met parfaitement au service de l'originalité cinglante et de l'absolue liberté de langage de Vian. Jimmy Walter se souvient :

Je crois avoir été le premier à mettre plusieurs tempos dans une même chanson. Dans "Les joyeux bouchers ", bien que l'on parle de tango, j'ai écrit une marche... ou j'écrivais un slow pour les couplets, et une valse pour le refrain, ce qui a plu à Boris, lui qui était anticonformiste. (Vian 2009, livret)

Ces deux premiers 45 tours sont rapidement suivis d'un projet de 33 tours, qui sortira sous le titre de Chansons possibles et impossibles pour lequel Vian fait désormais équipe avec un nouveau compositeur, arrangeur et pianiste, Alain Goraguer. Sur les quatre morceaux enregistrés en juin 1955, Vian fait le choix de réenregistrer « On n'est pas là pour se faire engueuler » et "Le petit commerce » dans de nouveaux arrangements de Goraguer dont l'approche stylistique et l'instrumentation sont plus ancrés dans la tradition du big band et servis cette fois par l'orchestre de Claude Bolling. Il enregistre également "Bourrée de complexe " et enfin "Je bois ", une ballade jazz qui présente des audaces harmoniques totalement inédites pour la chanson française. La mélodie et les modulations déroutantes de la partition de "Je bois » soulignent l'état de conscience altérée du personnage qui parle par la voix de Vian. 
Ainsi qu'il l'écrit un peu plus tard dans En avant la zizique... et par ici les gros sous, son essai sur l'industrie de la chanson, paru en 1958, Vian aime l'idée d'une chanson française nourrie de jazz (Vian 1966b, 168), mais il laisse entendre que les compositeurs français n'ont pas assez suivi l'évolution de cette musique. Il revient sur la question dans sa critique du premier album de Serge Gainsbourg en 1958 où il se réjouit de trouver de la " chanson jazz pas démodée pour la musique comme celles qu'on fait couramment en France dans l'esprit du jazz de 1935 (qui était parfait en 1935)! »(Verlant 1992, 64 ; italiques de Verlant). Le travail de Vian avec Jimmy Walter, Alain Goraguer et Henri Salvador sort la chanson de cette ornière et l'ouvre sur d'autres formes. Ainsi, à travers le jazz, s'opère-t-il dans la chanson de Vian une triple libération : celle d'un renouveau de la créativité musicale, celle d'une critique de l'autorité de l'État et enfin celle du langage poétique qui se transforme, sous l'impulsion de la syncope, d'un parti-pris d'inventivité et d'insolence. Voici ce que dira Serge Gainsbourg à propos de ces arrangements dans un entretien avec Noël Simsolo en 1984 :

Mais je repense au $25 \mathrm{~cm}$ de Vian... Les arrangements étaient d'un modernisme extraordinaire... Avec du jazz... Personne n'avait réussi à faire cela de façon aussi génialement sophistiquée. Sans compter l'aspect ‘ lyrics `, là c’est le plus grand... Avec moi bien sûr. [...] Vraiment son disque c'était une fixation pour moi... L'accompagnement musical ajoutait au tragique du texte... L'addition des mots dans "Les arts ménagers » ( La complainte du progrès ») c'est un collage dadaïste rehaussé par une musique terrifiante. Et le tout frappe très fort. (Simsolo 1984, 62)

\section{Le clin d'œil du musicien : un chapitre écrit comme un chorus de trompette}

Cet esprit du jazz qui anime l'écriture de chansons chez Vian accompagne l'ensemble de son œuvre. Largement commenté de ce point de vue, son roman le plus important, L'écume des jours, écrit en deux mois au cours de sa vingt-sixième année consacrait déjà la fusion entre la littérature et cette nouvelle musique née avec le siècle que Vian découvre à l'adolescence. Le projet qui consiste à transposer au roman certaines caractéristiques de la musique de jazz, s'effectue, dans L'écume des jours à plusieurs niveaux. Tout d'abord, celui d'un décor : il consiste par exemple, à introduire des noms de rues baptisées d'après de grands musiciens (la rue Sydney Bechet par exemple) ou à faire figurer dans le récit des situations multiples où il est question de musique de jazz, où les personnages en écoutent ou en parlent. "Êtes-vous arrangée par Duke Ellington ? " demande Colin en rencontrant Chloé pour la première fois. Ces choix esthétiques participent de la création d'un décor. Le travail stylistique qui fait intervenir une forme de prose poétique dans le récit, les marques de syncopes, les figures de synesthésie, ces procédés qui tentent d'imprégner le texte d'une essence jazz dont le lecteur ressentira l'expérience - peut-être à la façon dont Marcel Proust, sous l'influence de Ruskin, cherche à élever le texte au niveau de l'expérience même de l'œuvre d'art décrite (dans le fameux passage chez Mme de Saint-Euverte par exemple, cf. Proust 1954, 347-353) - ce 
travail-ci, ne relève plus du décor mais de la transposition d'une expérience esthétique à une autre par le biais du verbe. Par ailleurs, ainsi que Cécile Pajona le rappelait récemment, dans un article consacré au jazz littéraire dans L'écume des jours, la rapidité de composition et l'aspect relativement non-retravaillé du manuscrit témoignent, eux aussi, d'un procédé qui s'apparente à une approche jazz, musique de l'instant, qui naît dans le mouvement.

Nous tenterons, à présent, de distinguer un geste jazz d'une autre nature, dans L'écume des jours, qui n'a pas encore fait l'objet de commentaire et qui apparaît sous la forme d'un clin d'œil auto-référentiel, une sorte de inside joke semblable à celle de l'instrumentiste glissant dans son improvisation une citation que seule les initiés peuvent saisir au vol. Ici, Vian, le musicien s'adresse de façon cryptique aux musiciens, dans le chapitre 32. Pour toute personne pratiquant le jazz, le nombre 32 évoque la forme la plus répandue des morceaux, celle des chansons en 32 mesures, parmi les centaines que tous les jazzmen connaissent par cœur. Vian, très lié avec Raymond Queneau se montre oulipien avant l'heure en insérant dans ce chapitre 32, un texte qui semble épouser de manière troublante les contours d'un solo de jazz exécuté sur la forme standard d'une grille harmonique de 32 mesures. Il parsème son texte d'indices qui permettent de suivre cette référence à la trace, comme nous allons tenter de le montrer.

Dans le jazz, cette formule des 32 mesures concerne la majorité des standards du Great American Songbook constitué de mélodies souvent accompagnées d'une introduction et parfois d'une coda. Ces partitions en trente-deux mesures dans leur forme la plus courante sont fréquemment subdivisées, selon les sections de la chanson, et adoptent la progression suivante : couplet, couplet, pont, couplet, représentées par les lettres AABA. Parfois, le morceau est construit en parties notées ABAC, il y a bien entendu, de nombreuses variantes. Le chapitre 32 de L'écume des jours se présente sous la même forme dans toutes les éditions que nous avons consultées. C'est-à-dire que l'organisation des paragraphes suit celle du manus$\mathrm{crit}^{4}$. Une première phrase représente l'introduction, et est suivie de quatre paragraphes. Vian n'a semble-t-il pas cherché à mettre l'exercice trop en évidence. Comme dans l'art de la citation en jazz, il fait preuve d'élégance. Après une première lecture, où la phrase d'introduction se termine par un moment fort de percussion " comme un grand carton qu'on lâche à plat » qui suggère l'intervention d'un batteur ponctuant l'entrée du soliste s'élevant au-dessus de l'orchestre, ce qui frappe, dans la construction de ce chapitre 32, c'est la manière dont s'enchaînent les paragraphes et le changement de ton qui s'opère dans la partie centrale. En effet, au-delà de la concentration d'heptamètres signalée par Gilles Pestureau dans la préface à l'édition de 1994 (Vian 1994, 8) qui débute par " Mais Colin ne savait pas ", qui marque un changement de rythme, ce paragraphe central voit également s'opérer un changement de point de vue narratif, vers le monologue intérieur. Dans les standards du jazz organisés selon la structure AABA, il n'est pas inhabituel de changer de tonalité dans le pont. La progression harmonique et la mélodie de ce pont contrastent avec celle des couplets. Cette observation nous ayant mis sur la voie, on trouve ensuite d'autres éléments du texte qui, du point de vue du récit pur, pourraient sembler incongrus, mais qui dans la perspective d'une improvisation prennent un tout autre sens. Si, comme nous le supposons, Vian a choisi de mettre en scène 
la course de Colin comme la métaphore d'un musicien en pleine exécution d'un chorus, il a également choisi de placer des articulations dans le texte qui illustrent la façon dont un solo se construit et comment le musicien doit négocier certains passages car le travail d'improvisation est le résultat d'une composition mentale instantanée. Ainsi, la phrase, "il y avait encore trois rues avant la sienne " survient à la fin du premier A où le musicien commence à développer ses idées. On y trouve également le mot "sections » qui renvoie directement à l'organisation de la partition, mais aussi à la "section » des souffleurs dans un big band, qui intervient en réponse à l'improvisateur. Le paragraphe suivant fait apparaître Chloé, dans le récit. ${ }^{6}$ Les deux premiers paragraphes sont très équilibrés du point de vue de leur taille. Ils sont les deux A indissociables de la première partie du morceau, en parfaite harmonie l'un avec l'autre mais avec leurs caractères différents. Quant au troisième paragraphe, conformément à l'usage signalé plus haut, il présente une modulation. Les constructions heptasyllabiques marquent le glissement du discours narratif vers le monologue intérieur.

Le récit semble contenir un nouvel indice, laissé par Vian dans les phrases qui entament ce dernier paragraphe, le dernier A de la forme AABA : "le trottoir se dressa devant lui. Il le franchit d'un bond de géant. Il était au premier étage " à la sortie du pont, le musicien doit négocier élégamment l'intervalle harmonique qui le ramènera à la tonalité d'origine, ou signaler, s'il le souhaite, selon sa fantaisie, le retour dans la configuration de départ. Le "bond de géant » suggère peut-être un choix du musicien de passer au registre supérieur de son instrument, de jouer des notes hautes, comme il est souvent de mise pour terminer un solo en redoublant d'intensité. "Il était au premier étage " soulignerait ainsi que le musicien s'est élevé dans la tessiture de son instrument, mais aussi qu’il a regagné la partie A de la grille harmonique. En outre, si l'on s'accorde sur le fait qu'un chorus réussi présente souvent une progression vers un point culminant, on remarquera aussi qu'il s'achève, de coutume, par un atterrissage plus souple, où le musicien semble se poser après son envol. Comme l'indique " la paix des tapis ». Ainsi la sérénité de la fin «tout était calme et tranquille... elle était heureuse de le revoir » signifie la fin du chorus, avant le retour au début de la grille harmonique souligné par le mot " revoir »). Quant à cette phrase isolée de Nicolas "Ce n'est pas grandchose ", on peut se demander si Vian n'a pas voulu y dissimuler un dernier clin d'œil à Duke Ellington en proposant une formule qui évoque vaguement le célèbre "It Don't Mean a Thing ". En dernier lieu, ce chapitre 32 étant un des très rares chapitres de L'écume des jours où ne figure aucun échange de dialogues, il est possible d'y voir une indication supplémentaire de l'intention de Vian d'y représenter le parcours du musicien soliste, le temps d'un chorus sur trente-deux mesures.

Notre mise en avant de l'importance du jazz dans le processus créatif chez Vian nous a permis de mettre en évidence comment, par le biais de son travail de critique ou de celui de chanteur, celui-ci exprime à la fois des positions politiques sur l'actualité du monde, mais aussi vis-à-vis de questions politiques plus spécifiques aux acteurs du jazz. Son vif soutien du bebop, face aux critiques réactionnaires, démontre que selon sa perception, l'essence de cette musique réside dans le mouvement et la transformation. Si la musique de jazz naît de la réinvention d'un air, vouloir arrêter cet instinct transformatif, c'est vouloir en faire une mu- 
sique morte. "À bas la fossilisation! Moi j’aime entendre des choses que je ne connais pas » écrit-il en 1948 dans Jazz Hot (Vian 1971, 150). Aussi, Vian applique-t-il cette vision de l'art dans toute sa pratique créative. Le rapport étroit qu'il entretient avec le jazz lui permet de s'aventurer sur le terrain d'expérimentations formelles et d'accéder à une composition extrêmement stylisée du texte où se confondent procédés musicaux et poétiques. Son positionnement critique, son écriture de chansons et sa témérité littéraire, relèvent d'un même affect qui se manifeste par un geste jazz sans cesse réinventé.

\section{Notes}

1 L'intégrale de ses chroniques (parues dans des journaux comme Arts, Combat, Jazz-hot, Jazz Magazine, Jazz News ou encore inédites) ont été rassemblées en 1981 dans l'ouvrage Écrits sur le jazz. Un second opus, Autres écrits sur le jazz, est paru en 1982.

2 Voir également à ce propos Tournès 2001, 72-83.

3 Voir à ce sujet Constant-Martin/Roueff 2002, 170 citant pour exemple la critique de Louis Vuillemain, intitulée "Concerts métèques " (janvier 1923) dans le Courrier musical, qui accuse les amateurs de jazz, premières victimes d'une "machiavélique et empoisonnée propagande » de chercher à " gangréner notre organisme ".

4 La consultation du manuscrit de L'écume des jours en fac-similé par les éditions des Saints Pères (Vian 2013b) confirme cette organisation du texte. Nous remercions Cécile Pajona qui nous a gracieusement donné accès à ces pages.

5 Chorus , est le terme utilisé pour un solo qui s'étend sur un cycle complet de la grille harmonique, ici de 32 mesures.

6 Cette partie produit par ailleurs un effet similaire à celui d'un montage parallèle au cinéma. Deux scènes sont proposées consécutivement, mais liées du point de vue temporel et grammatical, comme le sont les deux premiers A de la partition.

\section{Bibliographie}

Arnaud, Noël : Les vies parallèles de Boris Vian. Paris : Bourgois, 1981.

Internationale Situationniste : "Avec et contre le cinéma ». In : Bulletin central édité par les sections de l'Internationale Situationniste 1 (Juin 1958), 8-9, http://www.labibliothequefantastique.net/ (consultation 10.12.18).

Bertolt, Nicole / Lapprand, Marc / Roulmann, François : Boris Vian : le swing et le verbe. Paris : Textuel, 2008.

Becker, Howard : Art Worlds. Berkeley/Los Angeles/London : University of California Press, 1982.

Constant-Martin, Denis / Roueff, Olivier : La France du jazz. Musique, modernité et identité dans la première moitié du XXème siècle. Marseille : Parenthèses, 2002. 
Fukagawa, Akiko : «La musique dans l'univers romanesque de L'écume des jours». In : Gallia 38 (1999), 41-48.

Lane, Jeremy F. : Jazz and Machine-Age Imperialism : Music, 'Race >, and Intellectuals in France, 1918-1945. Ann Arbor : University of Michigan Press, 2013.

Pajona, Cécile : «Vers une stylistique du jazz littéraire dans L'écume des jours de Boris Vian ». In : Musurgia 23,1 (2016), 105-122.

Panish, Jon : The Color of Jazz: Race and Representation in Postwar American Culture. Jackson : Univ. Press of Mississippi, 1995.

Pautrot, Jean-Louis : La Musique oubliée. Genève : Droz, 1994.

Proust, Marcel : À la recherche du temps perdu. Vol. 1. Paris : Gallimard, 1954.

Simsolo, Noël : "Serge Gainsbourg: ‘ J'ai pris la relève »». In : Dadoun, Roger et al. : Boris Vian (=L'Arc 90). Paris : Dhouailly, 1984, 62.

Tournès, Ludovic : "La réinterprétation du jazz : un phénomène de contreaméricanisation dans la France d'après-guerre (1945-1960) ». In : Revue française d'études américaines 5 (2001), hors-série, 72-83.

Vasseur, Nadine : "Jusqu'où va la zizique à Vian ». In : Dadoun, Roger et al. : Boris Vian (=L'Arc 90). Paris : Dhouailly, 1984, 59-60.

Verlant, Gilles : Gainsbourg. Paris : Albin Michel, 1992.

Vian, Boris: Troubles dans les Andains. Paris : La jeune Parque, 1966 .

Vian, Boris : En avant la zizique... et par ici les gros sous. Paris : La jeune Parque, 1966b.

Vian, Boris : Chroniques de jazz. Texte établi et présenté par Lucien Malson. Paris : 10/18, 1971.

Vian, Boris : Ecrits sur le jazz. Paris : Bourgois, 1981.

Vian, Boris : Autres écrits sur le jazz. Paris : Bourgois, 1982.

Vian, Boris : L'écume des jours. Éd. Gilbert Pestureau et Michel Rybalka. Paris : Bourgois, 1994.

Vian, Boris : L'écume des jours. Paris : Fayard/Pauvert, 2013a.

Vian, Boris : L'écume des jours. Manuscrit. Cambremer : Editions des Saints-Pères, 2013b.

Vihlen McGregor, Elizabeth : Jazz and Postwar French Identity Improvising the Nation. Lanham : Rowman and Littlefield, 2016.

\section{Discographie}

Gainsbourg, Serge : Du chant à la une ! Philips 76447, 1958 (LP).

Vian, Boris : Chansons possibles et impossibles. Philips 76042, 1956 (LP).

Vian, Boris : 100 chansons. Coffret Longbox. Productions Jacques Canneti DPVF28, 2009 (CD). 